\title{
Chaotic vibrations of closed cylindrical shells in a temperature field
}

\author{
A.V. Krysko ${ }^{\text {a }}$, J. Awrejcewicz ${ }^{\text {b,* }}$, E.S. Kuznetsova ${ }^{\mathrm{a}}$ and V.A. Krysko ${ }^{\mathrm{a}}$

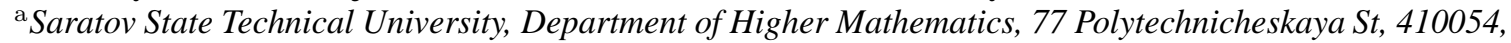 \\ Saratov, Russia \\ ${ }^{\mathrm{b}}$ Technical University of Lodz, Department of Automatics and Biomechanics, Stefanowskiego 1/15, 90-924 Lodz, \\ Poland
}

Received 2007

Revised 2007

Abstract. Complex vibrations of cylindrical shells embedded in a temperature field are studied, and the Bubnov-Galerkin method in higher approximations and in the Fourier representation is applied. Both lack and influence of temperature field on the shell dynamics are analyzed.

Keywords: Chaos, vibrations, shell, temperature field

\section{Formulation of the problem}

Problems related to the investigation of chaotic vibrations of flexible plates and shells attract attention of many engineers and applied mathematicians [1-10]. This is mainly motivated by an observation that harmful vibrations of plates and shells occur in various industry branches including space and aircraft factories.

In the frame of the nonlinear classical theory of shallow shells a closed cylindrical shell with circled cross section of finite length with both constant stiffness and density subjected to sign changeable loading and embedded into the temperature field is studied. Results of temperature field action are exhibited by shell buckling, which differs essentially from shell buckling yielded by mechanical load action. Thermal stresses occur owing to thermal extension of shell elements. Note that compression of the shell elements yields heat occurrence, whereas during shell elements extension a heat is absorbed. However, the frequently applied technical theory of shells does not introduce any differences between stresses produced directly by either temperature field or mechanical external loads.

The obtained solutions to the investigated problem may have an important impact for both rocket design industry or harmful effects occurred during transported cylinder-types containers fulfilled with fluids. In the first case, depending on the atmosphere conditions as well as on a moving rocket height, the external load acting on the rocket changes in time and may also change depending on the rocket surface. In the second case a loading of a moving container modeled by the cylindrical shell depends strongly on the movement parameters because sloshing of fluids occurs. Therefore, we study the classical problem but with inclusion of the shell loading depending both on time and coordinates $q=q(x, y, t)$. In addition, in a case of a moving rocket a key role plays a temperature field, what is taken into account of the investigated model.

The system of coordinates with $x$ axis coinciding with longitudinal coordinate, $y$ axis coinciding with a circle coordinate, as well as $z$ axis directed along a normal to the mean surface is introduced (Fig. 1). The cylindrical shell as a 3D object $\Omega$ is defined in the following way in the given system of coordinates: $\Omega=\{x, y, z \mid(x, y) \in$ $[0 ; L] \times[0 ; 2 \pi],-\leqslant z \leqslant h\}$.

\footnotetext{
${ }^{*}$ Corresponding author. E-mail: awrejcew $@$ p.lodz.pl.
} 


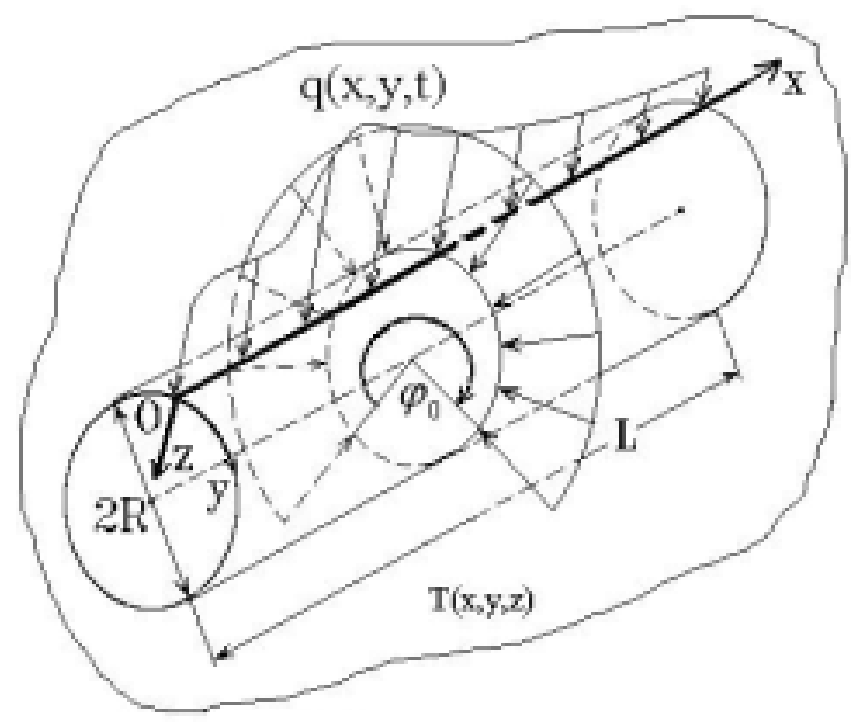

Fig. 1. Computation scheme.

The system of equations governing shell dynamics is presented in the following non-dimensional form [11]:

$$
\begin{aligned}
& \left\{\frac{1}{12\left(1-\nu^{2}\right)}\left[\lambda^{-2} \frac{\partial^{4} w}{\partial x^{4}}+2 \frac{\partial^{4} w}{\partial x^{2} \partial y^{2}}+\lambda^{2} \frac{\partial^{4} w}{\partial y^{4}}\right]-L(w, F)-k_{y} \frac{\partial^{2} F}{\partial x^{2}}-\frac{\partial^{2} w}{\partial t^{2}}-\varepsilon \frac{\partial w}{\partial t}-\right. \\
& \left.+\frac{1}{12\left(1-\nu^{2}\right)}\left(\lambda^{-1} \frac{\partial^{2} M_{t}}{\partial x^{2}}+\lambda \frac{\partial^{2} M_{t}}{\partial y^{2}}\right)\right\}+k_{y}^{2} q(x, y, t)=0, \\
& \left\{\lambda^{-2} \frac{\partial^{4} F}{\partial x^{4}}+2 \frac{\partial^{4} F}{\partial x^{2} \partial y^{2}}+\lambda^{2} \frac{\partial^{4} F}{\partial y^{4}}+\frac{1}{2} L(w, w)+k_{y} \frac{\partial^{2} w}{\partial x^{2}}+\lambda^{-1} \frac{\partial^{2} N_{t}}{\partial x^{2}}+\lambda \frac{\partial^{2} N_{t}}{\partial y^{2}}\right\}=0 .
\end{aligned}
$$

The following non-dimensional parameters are introduced (with bars):

$$
w=2 h \bar{w}, F=E_{0}(2 h)^{3} \bar{F}, t=\frac{R L}{2 h \sqrt{g E_{0}}} \bar{t}, \lambda=L / R ; x=L \bar{x}, y=R \bar{y} ; k_{y}=\bar{k}_{y} \frac{h}{R^{2}}, q=\bar{q} \frac{E_{0}(2 h)^{4}}{L^{2} R^{2}},
$$

where $L$ and $R=R_{y}$ are the shell length and radius, respectively, $t$ denotes time, $\varepsilon$ is the damping coefficient of a medium where the shell is embedded, $F$ is the stress (Airy's) function, $w$ denotes deflection, $h$ is the shell thickness, $\nu$ is Poisson's coefficient, $E_{0}$ is the Young modulus, $q(x, y, t)$ is the transversal load, $k_{y}$ is the shell curvature regarding $y$. In addition,

$$
L(w, F)=\frac{\partial^{2} w}{\partial x^{2}} \frac{\partial^{2} F}{\partial y^{2}}+\frac{\partial^{2} w}{\partial y^{2}} \frac{\partial^{2} F}{\partial x^{2}}-2 \frac{\partial^{2} w}{\partial x \partial y} \frac{\partial^{2} F}{\partial x \partial y}, L(w, w)=2\left[\frac{\partial^{2} w}{\partial x^{2}} \frac{\partial^{2} w}{\partial y^{2}}-\left(\frac{\partial^{2} w}{\partial x \partial y}\right)^{2}\right]
$$

are the known nonlinear operators,

$$
N_{t}=\frac{1}{h} \int_{-\frac{h}{2}}^{\frac{h}{2}} Q d z
$$

is the temperature-induced force,

$$
M_{t}=\frac{12}{h^{3}} \int_{-\frac{h}{2}}^{\frac{h}{2}} Q z d z
$$


is the temperature-induced moment, $Q(x, y, z)=T-T_{0}$ is the temperature increment, whereas $T_{0}$ is the initial temperature. For brevity of our considerations, bars over the non-dimensional quantities in Eq. (1) are omitted.

The system of Eq. (1) is supplemented by boundary and initial conditions, which are formulated below. The temperature field $T$ is given in the following form: $T(x, y)=C \sin (\pi x) \sin (\pi y)$.

Let us consider the dissipative system $(\varepsilon \neq 0)$ subjected to transversal loading distributed in zone $0 \leqslant \varphi \leqslant \varphi_{0}$, $0 \leqslant x \leqslant 1$ and being changed harmonically $q(t)=q_{0} \sin \left(\omega_{p} t\right)$, where $q_{0}$ and $\omega_{p}$ are the amplitude and frequency of the exciting force, respectively.

\section{The Bubnov-Galerkin method and Fourier representation}

The boundary value problem regarding space coordinates is solved by the Bubnov-Galerkin method in higher approximations. Functions $w$ and $F$ being solutions to Eq. (1) are approximated by an expression consisting of the product of functions depending on time and coordinates of the following form

$$
w=\sum_{i=0}^{N_{1}} \sum_{j=0}^{N_{2}} A_{i j}(t) \varphi_{i j}(x, y), F=\sum_{i=0}^{N_{1}} \sum_{j=0}^{N_{2}} B_{i j}(t) \psi_{i j}(x, y) .
$$

In order to find approximated values of functions $w$ and $F$ we take the coordinate systems of functions of the form $\left\{\varphi_{i j}(x, y), \psi_{i j}(x, y)\right\}(i, j=0,1,2 \ldots)$ in Eq. (2), which satisfy the following requirements:

1. $\varphi_{i j}(x, y) \in H_{A}, \psi_{i j}(x, y) \in H_{A}$, where $H_{A}$ is the Hilbert space, which is further referred to as the energy space.

2. $\forall i, j$ functions $\varphi_{i j}(x, y)$ and $\psi_{i j}(x, y)$ are linearly independent, continuous together with their up to the fourth order derivatives in space $\Omega$.

3. $\varphi_{i j}(x, y)$ and $\psi_{i j}(x, y)$ satisfy rigorously main boundary conditions (and initial conditions if any).

4. $\varphi_{i j}(x, y)$ and $\psi_{i j}(x, y)$ satisfy completeness property in $H_{A}$.

5. $\varphi_{i j}(x, y)$ and $\psi_{i j}(x, y)$ should represent $N$ first elements of a complete system of functions.

Coefficients $A_{i j}(t)$ and $B_{i j}(t)$ are the functions of time being sought. For convenience, the left hand sides of equations (1) given in brackets are denoted by $\Phi_{1}$ and $\Phi_{2}$, respectively, and therefore Eq. (1) takes the following form:

$$
\begin{aligned}
& \Phi_{1}\left(w, F, \frac{\partial^{2} w}{\partial x^{2}}, \frac{\partial^{2} F}{\partial x^{2}}, \ldots ; M_{t}, \frac{\partial^{2} M_{t}}{\partial x^{2}}, \frac{\partial^{2} M_{t}}{\partial y^{2}}\right)+k_{y}^{2} q(x, y, t)=0, \\
& \Phi_{2}\left(w, F, \frac{\partial^{2} w}{\partial x^{2}}, \frac{\partial^{2} F}{\partial x^{2}}, \ldots ; N_{t}, \frac{\partial^{2} N_{t}}{\partial^{2} x}, \frac{\partial^{2} N_{t}}{\partial^{2} y}\right)=0 .
\end{aligned}
$$

Applying the Bubnov-Galerkin procedure to Eq. (3) one gets

$$
\begin{aligned}
& \int_{0}^{1} \int_{0}^{\xi}{ }_{1} \varphi_{k l}(x, y) d x d y+\int_{x_{1}}^{x_{2}} \int_{y_{1}}^{y_{2}} k_{y}^{2} q(x, y, t) \varphi_{k l}(x, y) d x d y=0, \\
& \int_{0}^{1} \int_{0}^{\xi}{ }_{2} \psi_{k l}(x, y) d x d y=0, k=0,1, \ldots, N_{1} ; l=0,1, \ldots N_{2} .
\end{aligned}
$$

Now and further on we take $\xi=2 \pi$ for the closed cylindrical shell. Owing to Eq. (4), Eq. (3) take the following form

$$
\begin{aligned}
& \sum_{k l}\left[\sum_{i j} A_{i j} S_{i j r s k l}+\sum_{i j} B_{i j} C_{1, i j k l}+k_{y}^{2} Q_{k l}+H_{1 k l}-\sum_{i j} A_{i j} \sum_{r s} B_{r s} D_{1, i j r s k l}\right. \\
& -\sum_{i j}\left[\frac{d^{2} A_{i j}}{d t^{2}}+\varepsilon \frac{d A_{i j}}{d t}\right] G_{i j k l}=0, \\
& \sum_{k l}\left[\sum_{i j} A_{i j} C_{2, i j k l}+\sum_{i j} B_{i j} \sum_{r s} P_{i j r s k l}+\sum_{i j} A_{i j} \sum_{r s} A_{r s} D_{2, i j r s k l}+H_{2 k l}\right]=0 .
\end{aligned}
$$


Symbol $\sum_{k l}[*]$ before every equation of system Eq. (5) means that each of these equations is understood as the system of $k l$ equations of these types, and the associated Bubnov-Galerkin integrals have the following form

$$
\begin{aligned}
S_{i j s s k l} & =\int_{0}^{1} \int_{0}^{\xi} \frac{1}{12\left(1-\nu^{2}\right)}\left[\frac{1}{\lambda^{2}} \frac{\partial^{2} \varphi_{i j}}{\partial x^{2}} \frac{\partial^{2} \varphi_{r s}}{\partial x^{2}}+\lambda^{2} \frac{\partial^{2} \varphi_{i j}}{\partial y^{2}} \frac{\partial^{2} \varphi_{r s}}{\partial y^{2}}+2 \frac{\partial^{2} \varphi_{i j}}{\partial x \partial y} \frac{\partial^{2} \varphi_{r s}}{\partial x \partial x}\right] \varphi_{k l} d x d y, \\
C_{1, i j k l} & =\int_{0}^{1} \int_{0}^{\xi}\left[-k_{y} \frac{\partial^{2} \psi_{i j}}{\partial x^{2}}\right] \varphi_{k l} d x d y, \quad C_{2, i j k l}=\int_{0}^{\xi} \int_{0}^{1}\left[k_{y} \frac{\partial^{2} \varphi_{i j}}{\partial x^{2}}\right] \psi_{k l} d x d y, \\
D_{1, i j r s k l}= & \int_{0}^{1} \int_{0}^{\xi} L\left(\varphi_{i j}, \psi_{r s}\right) \varphi_{k l} d x d y, \quad D_{2, i j r s k l}=\int_{0}^{\xi} \int_{0}^{1} \frac{1}{2} L\left(\varphi_{i j}, \varphi_{r s}\right) \psi_{k l} d x d y, \\
P_{i j r s k l}= & \int_{0}^{1} \int_{0}^{\xi}\left[\frac{1}{\lambda^{2}} \frac{\partial^{2} \psi_{i j}}{\partial x^{2}} \frac{\partial^{2} \psi_{r s}}{\partial x^{2}}+\lambda^{2} \frac{\partial^{2} \psi_{i j}}{\partial y^{2}} \frac{\partial^{2} \psi_{r s}}{\partial y^{2}}+2 \frac{\partial^{2} \psi_{i j}}{\partial x \partial y} \frac{\partial^{2} \psi_{r s}}{\partial x \partial x}\right] \psi_{k l} d x d y, \\
G_{i j k l}= & \int_{0}^{1} \int_{0}^{\xi} \varphi_{i j} \psi_{k l} d x d y, \quad Q_{k l}=\int_{0}^{\xi} \int_{k l} \varphi_{k}(x, y, t) d x d y, \\
H_{1 k l}= & \int_{0}^{1} \int_{0}^{\xi} \frac{1}{12\left(1-\nu^{2}\right)}\left[\lambda^{-1} \frac{\partial^{2} M_{t}}{\partial x^{2}}+\lambda \frac{\partial^{2} M_{t}}{\partial y^{2}}\right] \varphi_{k l} d x d y, \\
H_{2 k l}= & \int_{0}^{1} \int_{0}^{\xi}\left[\lambda^{-1} \frac{\partial^{2} N_{t}}{\partial x^{2}}+\lambda \frac{\partial^{2} N_{t}}{\partial y^{2}}\right] \psi_{k l} d x d y .
\end{aligned}
$$

Integrals Eq. (6), except of $Q_{k l}$ corresponding only to the part of the shell area, are computed regarding the whole shell surface. After application of the Bubnov-Galerkin procedure the obtained system of ordinary differential equations with respect to functions $A_{i j}(t)$ and $B_{i j}(t)$ has the following matrix form

$$
\begin{aligned}
& \mathbf{G}(\ddot{A}+\varepsilon \dot{A})+\mathbf{S} A+\mathbf{C}_{1} B+\mathbf{D}_{1} A B=\mathbf{Q} q(t)+\mathbf{H}_{1}, \\
& \mathbf{C}_{2} A+\mathbf{P} B+\mathbf{D}_{2} A A=\mathbf{H}_{2},
\end{aligned}
$$

where: $\mathbf{G}=\left[G_{i j k l}\right], \mathbf{S}=\left[S_{i j r s k l}\right], \mathbf{C}_{1}=\left[C_{1 i j k l}\right], \mathbf{C}_{2}=\left[C_{2 i j k l}\right], \mathbf{D}_{1}=\left[D_{1 i j r s k l}\right], \mathbf{D}_{2}=\left[D_{2 i j r s k l}\right], \mathbf{P}=\left[P_{i j k l}\right]-$ square matrices of dimension $2 \cdot N_{1} \cdot N_{2} \times 2 \cdot N_{1} \cdot N_{2}$, and $\mathbf{A}=\left[A_{i j}\right], \mathbf{B}=\left[B_{i j}\right], \mathbf{Q}=\left[Q_{i j}\right]$ are the matrices of dimension $2 \cdot N_{1} \cdot N_{2} \times 1$.

Further, the second equation of system Eq. (7) is solved regarding matrix $\mathbf{B}$, and then it is solved by the method of inversed matrix on each time step:

$$
\mathbf{B}=\left[-\mathbf{P}^{-1} \mathbf{D}_{2} \mathbf{A}-\mathbf{P}^{-1} \mathbf{C}_{2}\right] \mathbf{A}+\mathbf{P}^{-1} \mathbf{H}_{2} \mathbf{C}_{2} A+\mathbf{P} B+\mathbf{D}_{2} A A=\mathbf{H}_{2} .
$$

Multiplying the first equation of Eq. (8) by $\mathbf{G}^{-\mathbf{1}}$ and introducing notation $\dot{\mathbf{A}}=\mathbf{R}$ the following Cauchy problem regarding nonlinear first order ODEs is formulated

$$
\left\{\begin{array}{l}
\dot{\mathbf{R}}=-\bar{\varepsilon} \mathbf{R}+\mathbf{G}^{-1} \mathbf{D}_{1} A B-\mathbf{G}^{-1} \mathbf{S} A+q(\bar{t}) \mathbf{G}^{-1} \mathbf{Q}+\mathbf{G}^{-1} \mathbf{H}_{1}, \\
\dot{A}=\mathbf{R} .
\end{array}\right.
$$

The introduced transformation is allowed since the inverse matrices $\mathbf{G}^{-1}$ and $\mathbf{P}^{-1}$ exist if the coordinate functions are linearly independent.

Equations (9) are supplemented by boundary and initial conditions and the obtained Cauchy problem is solved by the fourth-order Runge-Kutta method. A step in time is chosen via the Runge rule. Results given by various 
computational methods are compared in reference [12], where it is shown that integration by the fourth Runge-Kutta method is sufficient and the application of higher order Runge-Kutta approaches are time consuming and they do not yield improvement of the results.

\section{A numerical example}

In this section we study vibrations of a ball-type supported cylindrical shell along its edges and having the homogeneous boundary conditions [13]

$$
w=0 ; \quad \frac{\partial^{2} w}{\partial x^{2}}=0 ; \quad F=0 ; \quad \frac{\partial^{2} F}{\partial x^{2}}=0 \text { for } x=0 ; 1,
$$

and the following initial conditions

$$
\left.w(x, y)\right|_{t=0}=0,\left.\frac{\partial w}{\partial t}\right|_{t=0}=0 .
$$

In this case $\varphi_{i j}, \psi_{i j}$ in Eq. (2) are approximated by the product of two functions, where each depends only on one argument which satisfies boundary conditions Eq. (10):

$$
w=\sum_{i=1}^{N_{1}} \sum_{j=0}^{N_{2}} A_{i j}(t) \sin (i \pi x) \cos (j y), F=\sum_{i=1}^{N_{1}} \sum_{j=0}^{N_{2}} B_{i j}(t) \sin (i \pi x) \cos (j y) .
$$

The Bubnov-Galerkin algorithm described briefly so far allows a wide class of problems, both static and dynamic, to be solved. A solution to static problems is obtained via the set-up method first applied by Feodos'ev (the so called "set up method") [14], and widely applied for instance in monograph [5]. In order to solve static problems of plates and shells, various approximate methods have been applied allowing the partial differential equations to be reduced to the system of nonlinear algebraic equations, which is usually further linearized. In the set-up method a solution to PDEs is reduced to that of the Cauchy problem of ODEs. Results of static solutions to PDEs are reported and illustrated in reference [15].

Using the example of a closed cylinder type shell with parameters $k_{y}=112.5$ and $\lambda=2$, embedded in the temperature field and subjected to the following transversal load $q(t)=q_{0} \sin \left(\omega_{p} t\right)$ distributed on the whole shell surface $0 \leqslant \varphi \leqslant 2 \pi, 0 \leqslant x \leqslant 1$, for fixed loading amplitude $q_{0}=0.1$ we are going to study how the character of vibrations changes owing to the change of temperature intensity coefficient $C$ in relation $T(x, y)=$ $C \sin (\pi x) \sin (\pi y)$.

The fundamental characteristics such as signal $w(t)$, phase portrait $w\left(w^{\prime}\right)$, power spectrum $S\left(\omega_{p}\right)$ depending on the boundary conditions are shown in Table 1 . The values of $C$ are called threshold ones, since between the reported values of $C$ a picture is almost unchanged. Observe the following particularities of the shell behavior.

1. If $C=0$, then it means that there is no temperature field. The first row of the table exhibits harmonic shell vibrations. A phase portrait has the form of a circle and vibrations appear on the fundamental frequency: $\omega_{p}=\omega_{0}=26.1256$, where $\omega_{p}$ is the excitation frequency, which is clearly manifested in the frequency spectrum.

2. For $0<C \leqslant 24.2$ the temperature intensity increases, but the signal shape is not changed qualitatively, and vibrations are harmonic. The phase portrait exhibits a set of one rotated cycle.

3. Increasing the temperature field intensity by 0.04 , i.e. for $C=24.4$, the vibration character is changed, and the system transfers into chaos associated with an increase of deflection. The phase portrait is irregular, and power spectrum exhibits chaotic vibrations associated with both fundamental and its half frequencies.

4. In interval $24.4<C \leqslant 29$ changes appear in the vibration character, and from the broad band power spectrum "chaotic frequency part" is going to vanish.

5. For $C=30$ the signal is stabilized, and the phase plot exhibits a strange attractor. Power frequency spectrum consists of a linear combination of independent frequencies and bifurcations. A detailed analysis of the system behavior associated with the value of $C$ is given below. 
Table 1

Temperature amplitudes signals, phase portraits and power spectra of the cylindrical shell

0

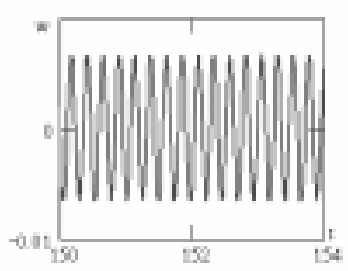

24.2

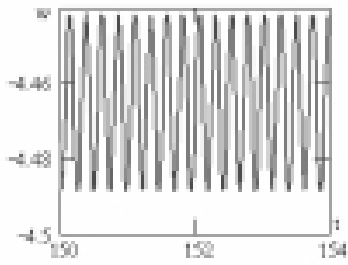

24.4

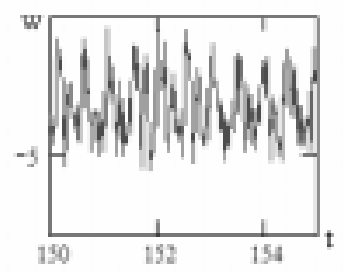

29

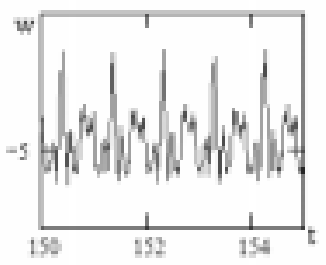

30

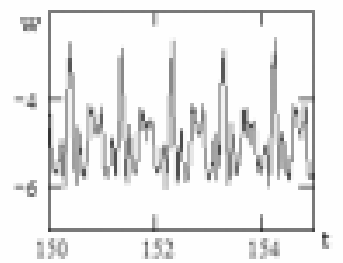

31

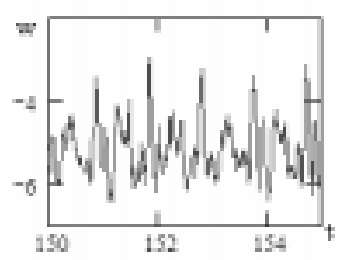

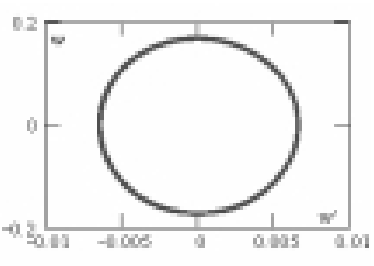
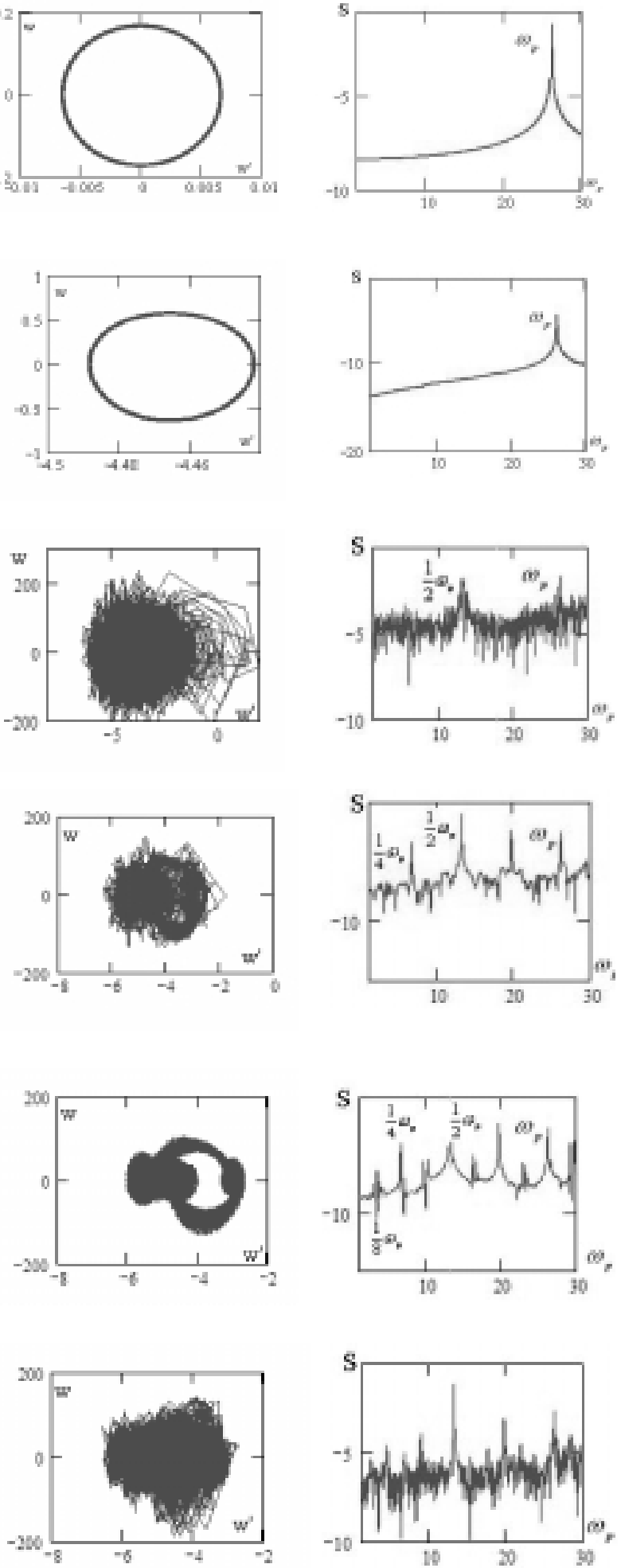
Table 2

Influence of variations in parameter $\left\{q_{0}\right\}$ for fixed $\mathbf{C}=30$

0.052

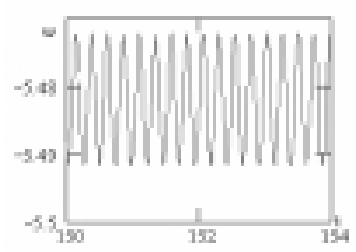

0.0522

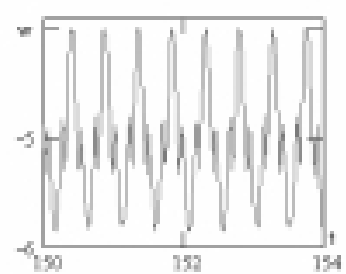

0.09

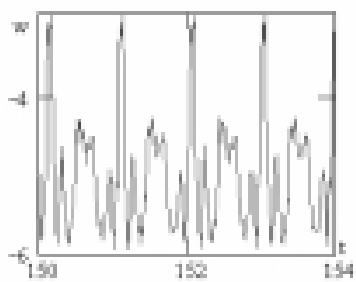

0.1

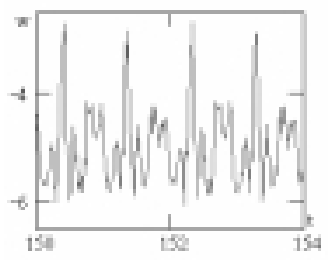

0.15

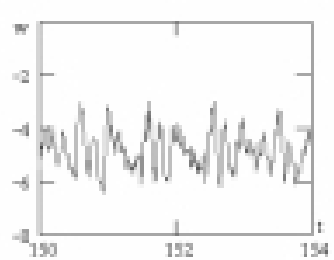

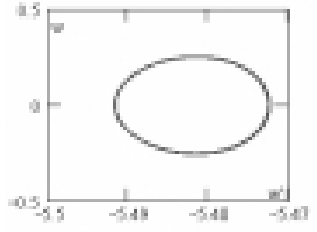
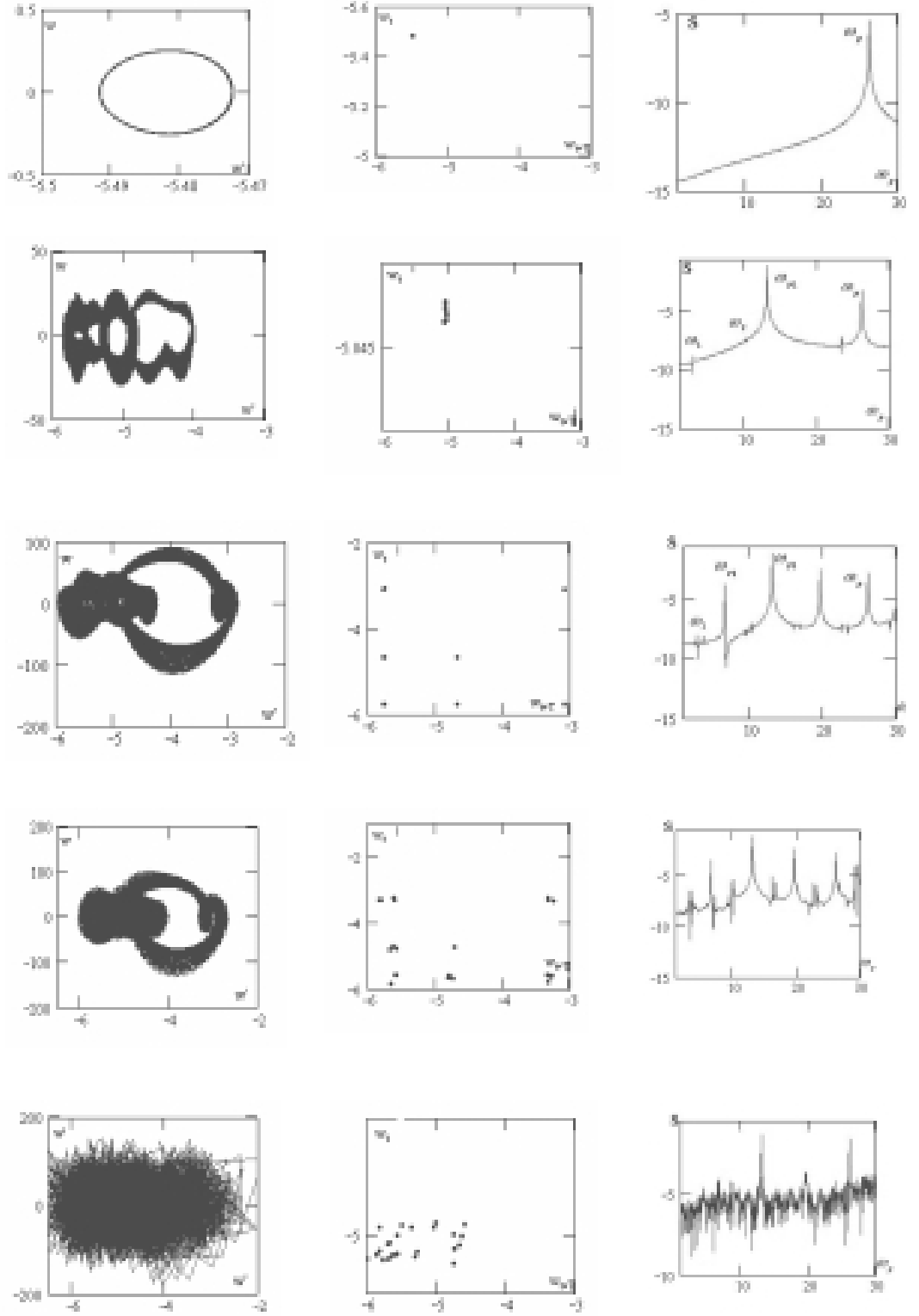

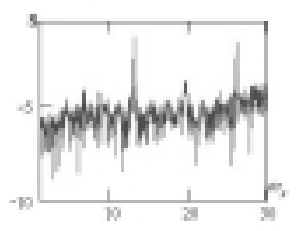


6. An increase of parameter $C$ intensity yields also the beam chaotic dynamics, however with different structure than that for $C=24.4$ since chaotic zones are mainly produced by period doubling bifurcations in this case.

Below, we will study the system behavior for fixed value of $C=30$ and for different values of external excitation amplitude $q_{0}$. The same characteristics as previously are applied as reported in Table 2.

The results presented in Table 2 are briefly summarized below.

1. For $q_{0}=0.052$ the beam exhibits periodic dynamics with the fundamental frequency $\omega_{0}$, and the Poincaré section clearly exhibits one point.

2. A slight variation of $q_{0}$ by the amount of $0.002\left(q_{0}=0.0522\right)$ changes qualitatively the system behavior. Namely, subharmonic vibrations appear with the fundamental frequency $\omega_{p 1}=\omega_{p} / 2=13.0628$, and a first independent frequency appears $\omega_{1}=2.8471$ producing the following linear combination $\omega_{2}=\omega_{p 1}-\omega_{1}=$ 10.2157 , i.e. quasi-periodic vibrations appear with two frequencies $\omega_{p 1}$ and $\omega_{1}$. Dobled-torus is also manifested in the phase projection.

3. For $q_{0}=0.09$ a period doubling bifurcation occurs $\omega_{p 2}=\omega_{p 1} / 2=6.5314$. In the Poincaré map four points appear, whereas the broadband structure of the phase portrait indicates occurrence of quasi-periodic vibrations.

4. A further increase of $q_{0}$ is associated with many period doubling bifurcations, and for $q_{0}>0.1$ the beam exhibits chaotic dynamics.

In other words, the described system behavior mainly concerns a transition from periodic and quasi-periodic dynamics to chaotic one.

\section{Concluding remarks}

Complex vibrations of the mechanical system represented by a closed cylindrical shell with a circled cross section and of finite length are studied in this work. The investigations are carried out using the Bubnov-Galerkin method with Fourier representation and with higher approximation regarding spatial coordinates.

The novel scenario of the studied shell transition from periodic to chaotic vibrations via the collapse of quasiperiodic vibrations with one independent frequency and period doubling bifurcation is illustrated and discussed. Analysis of the system behavior with the change of parameter $C$ (temperature intensity) in the formula $T(x, y)=$ $C \sin (\pi x) \sin (\pi y)$ and for the fixed value of $q_{0}=0.1$ (Table 1) as well as for fixed value of $C=30$ (Table 2) has been carried out. Additionally, it is shown (Table 2) how for various intensities of the temperature field, including its lack, an increase of the loading induces qualitative changes in the investigated shell dynamics, and how chaotic zones are transmitted into harmonic ones and vice versa.

\section{References}

[1] V.S. Avduyevskiy, B.M. Galitseyskiy and G.A. Glebov, Introduction to Heat Transfer in Airplanes and Rocket-Cosmic Techniques, Avduyevskiy, Koshkin, Mashinostroyeniye, Moscow, 1992, in Russian.

[2] J. Awrejcewicz and V.A. Krysko, Feigenbaum scenario exhibited by thin plate dynamics, Nonlinear Dynamics 24 (2001), $373-398$.

[3] J. Awrejcewicz, V.A. Krysko and A.V. Krysko, Spatio-temporal chaos and solitons exhibited by von Kármán mode, International Journal of Bifurcation and Chaos 12(7) (2002), 1465-1513.

[4] J. Awrejcewicz and A.V. Krysko, Analysis of complex parametric vibrations of plates and shells using Bubnov-Galerkin approach, Archive of Applied Mechanics 73 (2003), 495-504.

[5] J. Awrejcewicz and V.A. Krysko, Nonclassic Thermoelastic Problem in Nonlinear Dynamics of Shells, Springer-Verlag, Berlin, 2003.

[6] J. Awrejcewicz, V.A. Krysko and A.F. Vakakis, Nonlinear Dynamics of Continuous Elastic Systems, Springer-Verlag, Berlin, 2004.

[7] V.M. Bakulin, I.F. Obraztsov and V.A. Potopakhin, Dynamical Problems of Theory of Composite Shells, Influence of Thermo-Dynamic Loads and Concentrated Energy Flows, Moscow, FizMatLit, 1998, in Russian.

[8] V.A. Krysko, J. Awrejcewicz and T.V. Shchekaturova, Chaotic vibrations of spherical and conical axially-symmetric shells, Archive of Applied Mechanics 74(5-6) (2005), 338-358.

[9] V.A. Krysko and I.V. Kravtsova, Control of Chaotic Vibrations of Flexible Spherical Shells, Izvestia RAS, Mekhanika Tverdogo Tela, 1, 2005, pp. 140-150, in Russian.

[10] J. Awrejcewicz, V.A. Krysko and A.V. Krysko, Thermo-Dynamics of Plates and Shells, Springer-Verlag, Berlin, 2007.

[11] A.S. Volmir, Stability of Elastic Systems, Moscow, Fizmatgiz, 1963, in Russian. 
[12] V.A. Krysko and G.G. Narkaytis, Comparison of Various Computational Methods using Example of Vibration Modelling of Flexible Infinite Plates Subjected to Sign Changeable Loads, Proceedings of XXI International Conference on Plate and Shell Theories, Saratov, 2005, pp. 281-288.

[13] M.S. Kornishin, Nonlinear Problems of Plates and Shallow Shells and Methods of Their Solution, Moscow, Science, 1964, in Russian.

[14] V.I. Feodos'ev, On the method of solution of stability of deformable systems, Prikladnaya Matematika i Mekhanika 27(2) (1963), 265-275, in Russian.

[15] V.A. Krysko, N.E. Saveleva and K.F. Shagivaleev, Statics and dynamics of closed cylindrical shells subjected to non-uniform transversal loading, Izvestia VUZ, Mashinostroyeniye 1 (2005), 3-14, in Russian. 

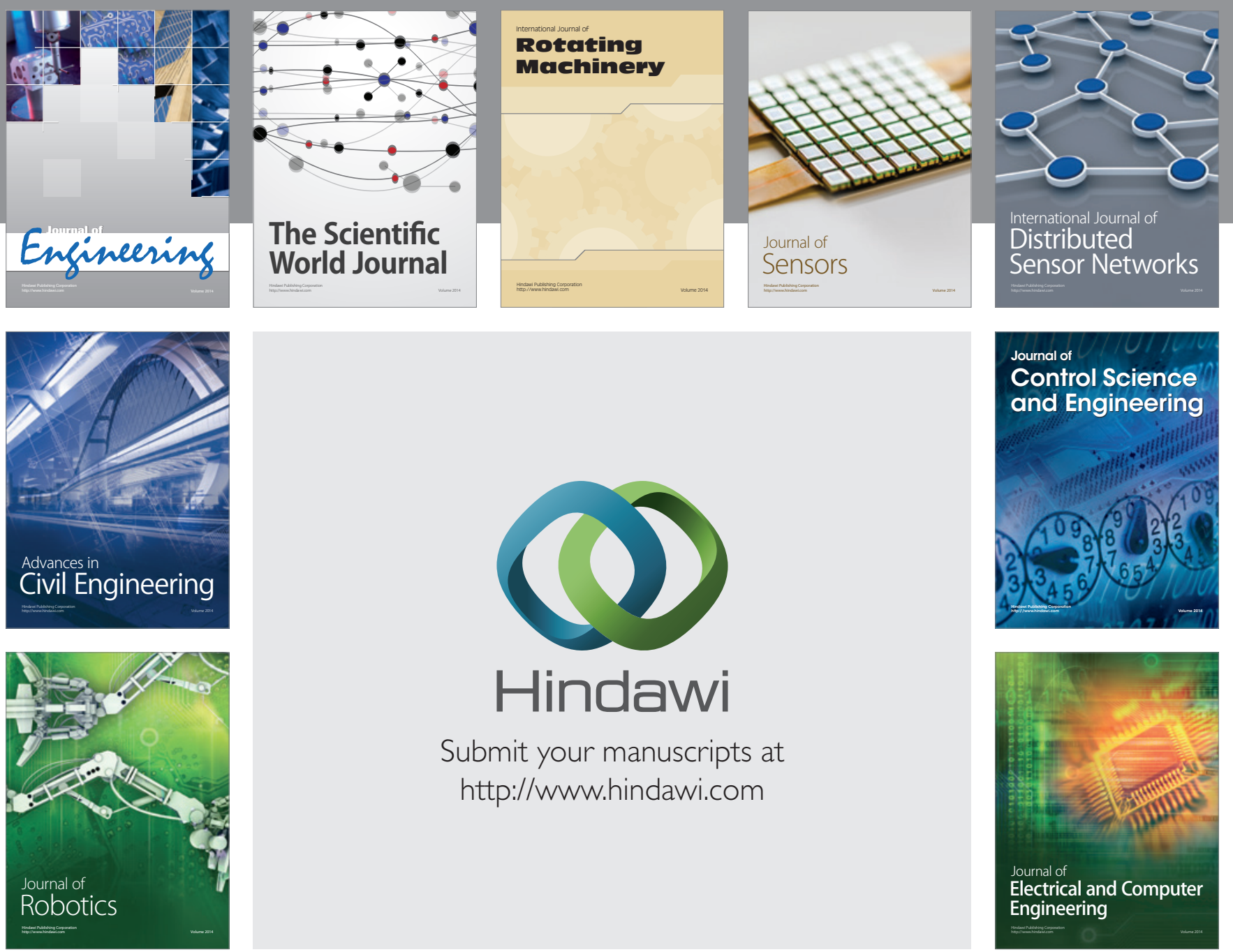

Submit your manuscripts at

http://www.hindawi.com
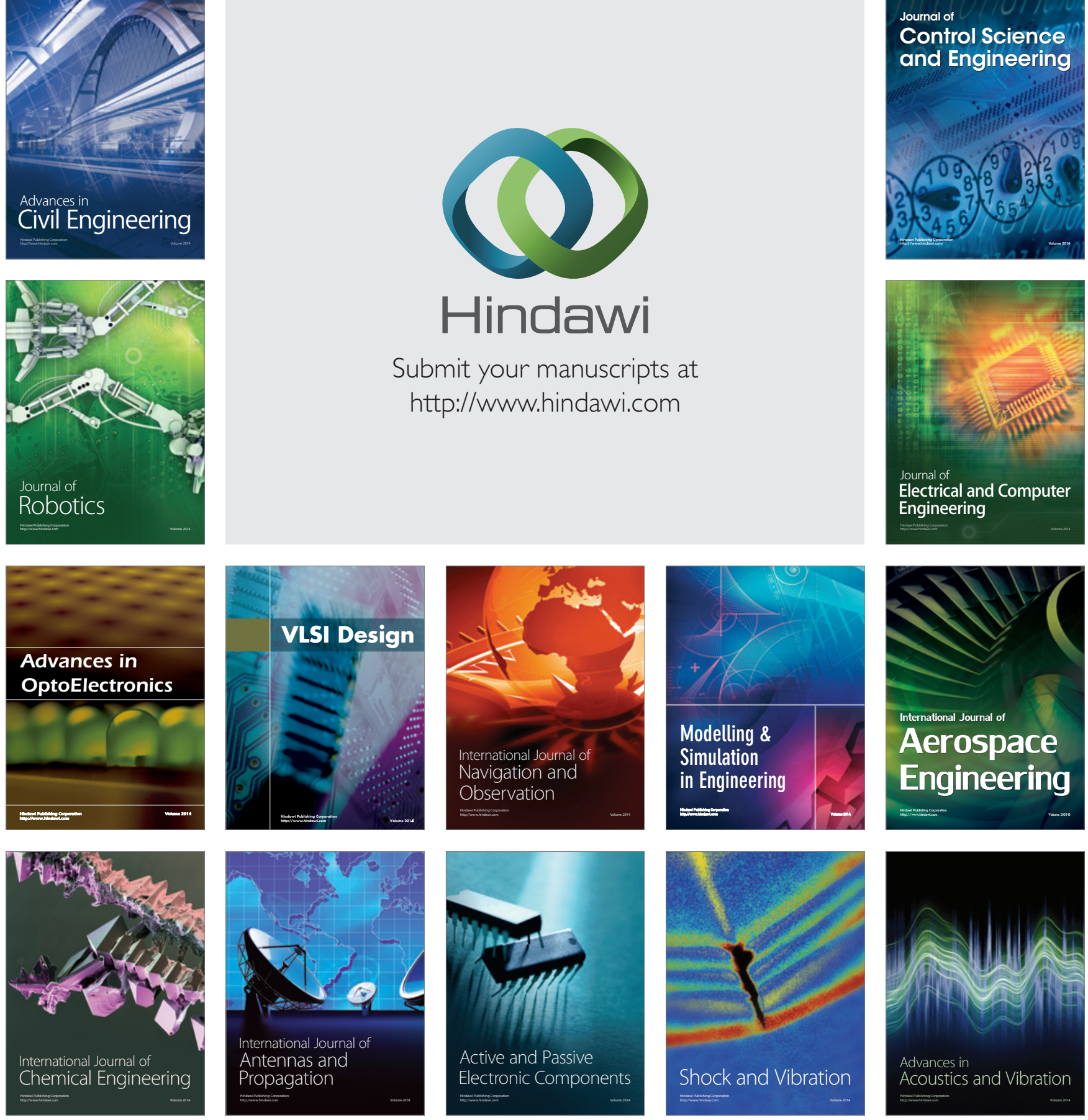